\title{
Effect of nonmesogenic impurities on the liquid crystalline phase transitions of octylcyanobiphenyl
}

\author{
Katleen Denolf, George Cordoyiannis, Christ Glorieux, and Jan Thoen* \\ Laboratorium voor Akoestiek en Thermische Fysica, Departement Natuurkunde en Sterrenkunde, Katholieke Universiteit Leuven, \\ Celestijnenlaan 200D, bus 2416, B-3001 Leuven, Belgium
}

(Received 17 July 2007; published 7 November 2007)

\begin{abstract}
The effect of the nonmesogenic solutes cyclohexane $(\mathrm{CH})$, biphenyl $(\mathrm{BP})$, and water $(W)$ on the nematicisotropic $(N-I)$ and the nematic-smectic- $A(N-\mathrm{Sm} A)$ phase transitions in the liquid crystal octylcyanobiphenyl (8CB) has been studied by means of adiabatic scanning calorimetry. For BP and $\mathrm{CH}$ a linear decrease of both transition temperatures was observed with increasing solute mole fraction. For water the transition temperature stayed nearly constant (after a slight decrease for mole fractions of water up to 0.1) over the entire investigated region. For all concentrations of biphenyl and water studied the $N-\operatorname{Sm} A$ transition remains second order. For $8 \mathrm{CB}+\mathrm{CH}$, however, crossover from second order to first order is observed at a tricritical point of the mole fraction $x$ of $\mathrm{CH}$ around 0.046 . For water it was found that for mole fractions of water above 0.1 phase separation between an $8 \mathrm{CB}$ rich and a water rich phase occurred. The changes in the transition temperatures and differences between the three systems, and in particular the crossover from second order to first order in the $8 \mathrm{CB}+\mathrm{CH}$ system, is explained in terms of a mean-field free energy density expression including coupling terms of the solutes mole fraction with the nematic and smectic- $A$ order parameters.
\end{abstract}

DOI: 10.1103/PhysRevE.76.051702

PACS number(s): 64.70.Md, 65.20.+w

\section{INTRODUCTION}

Liquid crystalline materials can exhibit a wide variety of mesophases with symmetry intermediate between that of solid crystals and of isotropic liquids. Such liquid crystalline phases possess orientational order but no or reduced positional order [1,2]. The many different phases and phase transitions make liquid crystals good model systems for testing general phase transition and critical phenomena concepts. In particular, the first-order or second-order (or continuous) character of the transition and the universality class of the critical exponents has been extensively investigated by many different techniques.

High resolution adiabatic and ac calorimetric techniques have contributed substantially by revealing subtle thermal features and fluctuation effects at the phase transitions [3-5]. In particular adiabatic scanning calorimetry has proven to be an important tool to discriminate between first-order and second-order transitions in addition to render high-resolution information on pretransitional specific heat capacity behavior.

The most common phase transition is the one between the isotropic $(I)$ liquid phase and the nematic $(N)$ phase possessing orientational but no positional order. This transition is weakly first order [1,2] with small latent heats and quite often substantial pretransitional fluctuation effects [6].

However, one of the most interesting and most extensively studied transitions is the nematic $(N)$ to smectic- $A$ $(\operatorname{Sm} A)$ phase transition [7]. In addition to orientational order the $\operatorname{Sm} A$ phase exhibits one-dimensional positional order. This positional order of the $\operatorname{Sm} A$ phase can be described in terms of a two-component complex order parameter, and thus the $N-\operatorname{Sm} A$ transition was expected to be in the three dimensional (3D) $X Y$ universality class [8]. However, experi-

\footnotetext{
*jan.thoen@fys.kuleuven.be
}

ments have revealed nonuniversal critical behavior and anisotropic divergences of correlation lengths [7]. In addition to that, coupling with the nematic order parameter [1] and nematic fluctuations may affect the order of the transition. It has been shown by de Gennes $[1,8]$ that strong coupling (narrow nematic range) between the $N$ and $\operatorname{Sm} A$ order parameters results in a first-order $N-\operatorname{Sm} A$ transition, while weak coupling (wide nematic range) gives a continuous transition. High-resolution adiabatic scanning calorimetry (ASC) results on mixtures of alkylcyanobiphenyls (nCBs) are consistent with this picture [9]. However, it was predicted by Halperin et al. [10] that coupling between the $\operatorname{Sm} A$ order parameter and the nematic director fluctuations may drive an otherwise continuous transition to a (weakly) first-order one. Two compounds for which this aspect was investigated in detail are 8CB (octylcyanobiphenyl) [11] and 8OCB (octyloxycyanobiphenyl) [12]. No evidence for a first-order latent heat could be found within an upper limit of $1.4 \mathrm{~J} / \mathrm{kg}$ for $8 \mathrm{CB}$ and of $1.8 \mathrm{~J} / \mathrm{kg}$ for $8 \mathrm{OCB}$. However, optical investigations showed for $8 \mathrm{CB}$ some evidence for a very small two-phase region and a very small discontinuity in the $\operatorname{Sm} A$ order parameter at the $N-\operatorname{Sm} A$ transition [13]. Estimates for a latent heat, if present, at the $N-\mathrm{Sm} A$ transition of $8 \mathrm{CB}$, induced by the latter coupling, are apparently smaller than the upper limit obtained from ASC [14].

It was also suggested theoretically $[15,16]$ that for chiral compounds the transition between the cholesteric or chiral nematic $\left(N^{*}\right)$ phase and the smectic- $A$ phase should also be first order. This prediction was experimentally confirmed by an investigation of mixtures of the nonchiral compound octyloxycyanobiphenyl (8OCB) (a compound with a continuous $N-\operatorname{Sm} A$ transition) and the chiral 4-(2-methylbutyl)4 '-cyanobiphenyl (CB15) [12].

Another type of coupling that potentially could drive the $N-\operatorname{Sm} A$ transition from second order to first order is the coupling with a nonmesogenic (impurity) solute, which has been occasionally invoked theoretically but never studied experi- 
mentally [17-19]. In order to investigate this possible coupling effect we carried out ASC measurements on a series of binary mixtures of the liquid crystal $8 \mathrm{CB}$ with three nonmesogenic solutes: cyclohexane $(\mathrm{CH})$, biphenyl (BP) (a molecule identical to the aromatic core of $8 \mathrm{CB}$ ), and water $(W)$. A brief account of results for the $N-\operatorname{Sm} A$ transitions of the biphenyl-8CB and cyclohexane-8CB systems has been given in a recent paper [20] where also an explanation in terms of a mean-field free energy density expression was put forward. Here we give full details of these investigations including additional measurements for the cyclohexane- $8 \mathrm{CB}$ system and for a third system, water-8CB.

\section{THEORETICAL BACKGROUND}

The thermodynamic behavior near the nematic-isotropic transition $(N-I)$ is usually described in terms of the meanfield Landau-de Gennes theory [1]. For the uniaxial nematic phase one obtains an expansion of the free energy density $F$ in terms of the nematic order parameter $\mathrm{S}$ :

$$
F-F_{I}=\frac{1}{2} A_{0} S^{2}-\frac{1}{3} B_{0} S^{3}+\frac{1}{4} C_{0} S^{4}+\cdots .
$$

In the isotropic phase $S=0$ and in the nematic phase $S \neq 0$. In Eq. (1) one has $A_{0}=a\left(T-T^{*}\right)$ (with $a>0$ ) and $B_{0}>0$ for uniaxial phases with positive anisotropy. The presence of the cubic term that does not disappear at $T_{N-I}$, leads to a firstorder transition with a finite discontinuity in the order parameter $\left[S_{N-I}=2 B_{0} /\left(3 C_{0}\right)\right] . T^{*}$ is the lower stability limit of the isotropic phase. The nonzero value of $B_{0}$ results in a (typically quite small) enthalpy discontinuity (latent heat) at $T_{N-I}$,

$$
\Delta H_{L}=H_{I}-H_{N}=\frac{2 a B_{0}^{2}}{9 C_{0}^{2}} T_{N-I} .
$$

Since the pure $8 \mathrm{CB}$ liquid crystal has a continuous $\mathrm{N}-\mathrm{Sm} A$ transition with an effective specific heat capacity critical exponent $\alpha$ value between the three-dimensional (3D) $X Y$ value and the tricritical value $[7,11]$, the expression of the free energy density for this transition should include the Landau-de Gennes coupling between the $N$ order parameter $S$ and the $\operatorname{Sm} A$ order parameter amplitude $\psi[1,8]$,

$$
F=F_{N}(S)+\frac{1}{2} \alpha(T) \psi^{2}+\frac{1}{4} \beta \psi^{4}-C \psi^{2} \delta S+\frac{1}{2 \chi} \delta S^{2}+\cdots .
$$

$F_{N}$ is the nematic free energy density given by Eq. (1). For $T<T_{N-S m A}$, one has $\delta S=S-S_{0}$, with $S_{0}(T)$ the nematic order parameter in the absence of smectic order. The temperature dependence of $\alpha$ is given by $\alpha(T)=\alpha_{0}\left(T-T_{0}\right)$, and $\chi(T)$ is a response function that is large near $T_{N-I}$ but decreases with decreasing temperature. $\alpha_{0}, \beta$, and $C$ are positive constants. Minimization of $F$ with respect to $\delta S$ gives

$$
\delta S=C \psi^{2} \chi
$$

Substitution of Eq. (4) in Eq. (3) results in

$$
F=F_{N}(S)+\frac{1}{2} \alpha(T) \psi^{2}+\frac{1}{4} \beta^{\prime} \psi^{4}+\cdots,
$$

with

$$
\beta^{\prime}=\beta-2 C^{2} \chi
$$

Depending on the values of $C$ and $\chi$ and the resulting sign of $\beta^{\prime}$ three different cases can be obtained. For $\beta^{\prime}>0$ one has a continuous transition with $T_{0}=T_{N \text {-SmA } A}, \beta^{\prime}<0$ corresponds to a first-order transition, and at $\beta^{\prime}=0$ one has a crossover from a (second-order) continuous transition to a first-order transition at a tricritical point.

The addition of nonmesogenic impurities to $8 \mathrm{CB}$ affects both the $N-I$ transition as well as the $N-\operatorname{Sm} A$ transition. Elements (see Sec. IV) that must be understood are as follows. For both transitions the decrease of the transition temperatures with solute mole fraction $x$ must be understood. For the $N-I$ transition the almost mole fraction independent latent heats (in the investigated mole fraction range) must be considered. For the $N-\operatorname{Sm} A$ transition the crossover (with increasing $x$ ) from second order to first order for $8 \mathrm{CB}+\mathrm{CH}$ and the absence of it for $8 \mathrm{CB}+\mathrm{BP}$ and $8 \mathrm{CB}+W$ must be explained. Let us first look at the $N-\operatorname{Sm} A$ transition. Considering different possibilities, it turned out that the following modification of Eq. (3) with two mole fraction $x$ dependent terms is appropriate

$$
\begin{aligned}
F= & F_{N}(S)+\frac{1}{2} \alpha \psi^{2}+\frac{1}{4} \beta \psi^{4}-C \psi^{2} \delta S+\frac{1}{2 \chi} \delta S^{2}+A \psi^{2} x \\
& +D \psi^{2} \delta S x+\cdots .
\end{aligned}
$$

Again minimizing with respect to $\delta S$ and substitution in Eq. (7) gives

$$
\begin{gathered}
\delta S=(C-D x) \psi^{2} \chi, \\
F=F_{N}(S)+\frac{1}{2} \alpha^{\prime}(T) \psi^{2}+\frac{1}{4} \beta^{\prime \prime} \psi^{4},
\end{gathered}
$$

with

$$
\begin{gathered}
\alpha^{\prime}=\alpha+2 A x, \\
\beta^{\prime \prime}=\beta^{\prime}+4 D C \chi x=\beta-2 C^{2} \chi+4 D C \chi x, \\
T_{N-\operatorname{Sm} A}(x)=T_{N-\operatorname{Sm} A}(0)-\frac{2 A x}{\alpha_{0}} .
\end{gathered}
$$

As it can be seen from Eq. (12), a coupling term with $A$ $>0$ would be needed to obtain a (linear) decrease of the $N$-Sm $A$ transition temperature with increasing $x$. A possible change of the order of the transition via the renormalization of $\beta$ in Eq. (3) can be accounted for by the $x$-dependent term in Eq. (11). For $\beta^{\prime}>0$ (as in the case of pure 8CB), $D$ would have to be negative to accomplish a transition from secondorder to first-order.

In order to arrive at a (linear) transition temperature change for the $N-I$ transition with increasing $x$, Eq. (1) must be extended also with a coupling term between $S$ and $x$ of the form $E S^{2} x$. This would result in 


$$
\begin{gathered}
a(x)=\frac{a}{2}+E x, \\
T_{N-I}(x)=T_{N-I}(0)-\frac{2 E x}{a} .
\end{gathered}
$$

Assuming that $E$ is a constant would result in a first-order approximation in a mole fraction independent latent heat given by Eq. (2). A coupling term between the mole fraction $x$ and the nematic order parameter $S$ also has a (small) effect on the renormalized $\beta$ expression in Eq. (11). Adding in Eq. (7) a term $E S^{2} x=E\left(\delta S+S_{0}\right)^{2} x$ and minimization with respect to $\delta S$ results in

$$
\delta S=\chi^{\prime}\left(C \psi^{2}-D \psi^{2} x-2 E S_{0} x\right),
$$

with

$$
\chi^{\prime}=\frac{\chi}{1+2 E S_{0} x} .
$$

Substitution in the adjusted Eq. (7) (with the additional term $E S^{2} x$ ) and further assuming $\left(\chi^{\prime}\right)^{2} / \chi \approx \chi^{\prime}$ results (to lowest order in $x$ ) in

$$
\begin{gathered}
\alpha^{\prime \prime}=\alpha+2 A x\left(1+\frac{2 C E S_{0} \chi^{\prime}}{A}\right), \\
\beta^{\prime \prime \prime}=\beta-2 C^{2} \chi^{\prime}+4 D C \chi^{\prime} x\left(1+4 E C \chi^{\prime}\right), \\
T_{N-\operatorname{Sm} A}(x)=T_{N-\operatorname{Sm} A}(0)-\frac{2 A x}{\alpha_{0}}\left(1+\frac{2 C E S_{0} \chi^{\prime}}{A}\right) .
\end{gathered}
$$

For decreasing $N-I$ transition temperatures $(E>0)$ with increasing $x$ (as the case for our systems), the fact that $\chi^{\prime}<\chi$ reduces the second term in Eq. (18) in comparison with the corresponding one in Eq. (11) and thus takes the transition somewhat further away from the tricritical point on the second-order side of the phase transition line as a function of mole fraction.

\section{EXPERIMENT}

In order to study the effect of nonmesogenic impurities on the liquid crystalline phase transitions of octylcyanobiphenyl (8CB) we carried out adiabatic scanning calorimetry measurements. Adiabatic scanning calorimetry is designed to obtain continuously the evolution of the heat capacity $C_{p}$ and the enthalpy $H$ of a sample of interest as a function of temperature $[3,4]$. Both quantities can be studied with a very high precision and temperature resolution while maintaining thermal equilibrium inside the sample. To obtain $C_{p}$ and $H$, a constant power $P$ is supplied to the sample and the resulting change in temperature $T(t)$ is measured as a function of time. From these two quantities, the heat capacity can be easily calculated,

$$
C_{p}=\frac{P}{\dot{T}(t)},
$$

with $\dot{T}(t)=d T / d t$ the time derivative of the temperature, to be calculated numerically. Besides information about $C_{p}$, the exact knowledge of $T(t)$ allows one also to calculate directly the enthalpy $H$ as a function of temperature by inverting the $T(t)$ data to

$$
H(T)=H\left(T_{0}\right)+P\left[t(T)-t\left(T_{0}\right)\right],
$$

with $T_{0}$ the starting temperature of the run. The continuous determination of the enthalpy as a function of temperature provides a unique tool for determining the order of the phase transition: if the enthalpy shows a jump at a certain temperature (i.e., a latent heat is present), the transition is first-order; if such a jump is absent, the transition is continuous (or second-order). In case of a broadened first-order transition, the two-phase region can be clearly identified. The fact that the direct ASC result is $H(T)$ allows one to define for secondorder transitions the quantity

$$
C=\frac{H-H_{c}}{T-T_{c}},
$$

with $H, H_{c}$ being the enthalpy at the temperature $T$ and at the critical temperature $T_{c}$, respectively. If the limiting specific heat capacity is of the form

$$
C_{p}=A^{ \pm}|\tau|^{-\alpha}+B
$$

with $\tau=\left(T-T_{c}\right) / T_{c}, A^{ \pm}$the critical amplitudes above and below $T_{c}, \alpha$ the critical exponent, and $B$ the background term, it can be shown that [11]

$$
C-C_{p}=\frac{A^{ \pm}}{1-\alpha}|\tau|^{-\alpha}
$$

Thus, a $\log -\log$ plot of $C-C_{p}$ versus $\tau$ shows a linear behavior with slope $-\alpha$.

In this paper we present high-resolution ASC results of 8CB diluted with three different nonmesogenic solutes: cyclohexane $(\mathrm{CH})$, biphenyl $(\mathrm{BP})$, and water $(W)$. Cyclohexane (purity better than 99.9\%) was obtained from VEL, Leuven, Belgium; biphenyl (purity better than 99.5\%) was obtained from Sigma-Aldrich Chemie, Germany; and for the water mixtures we used triple distilled water. For the 8CB compound two different batches were used. The old (1999) batch 1 with purity of $99.9 \%$ was obtained from the laboratory of Dabrowski (Military University of Technology, Warsaw, Poland) and used initially for five mixtures (with mole fractions $x_{\mathrm{CH}}$ of $0.0104,0.0183,0.0368,0.0467$, and 0.0722 ) of $8 \mathrm{CB}$ $+\mathrm{CH}$. For the other $8 \mathrm{CB}+\mathrm{CH}$ mixtures and the subsequent measurements of $8 \mathrm{CB}+\mathrm{BP}$ and $8 \mathrm{CB}+W$ mixtures a new (2006) high purity (better than 99.9\%) batch 2 of 8CB, also synthesized at the laboratory of Dabrowski, was used. A tantalum cylindrical cell with a diameter of $10 \mathrm{~mm}$ and a length of $50 \mathrm{~mm}$ was used. The cell contained a stirring ball allowing continuous stirring in the usual way $[3,4]$ by rolling the ball back and forth from one end to the other end of the cell by changing the inclination of the horizontally suspended cell. Sample masses in the cell were typically between 1.5 and $2.0 \mathrm{~g}$. Both heating and cooling runs were executed. 


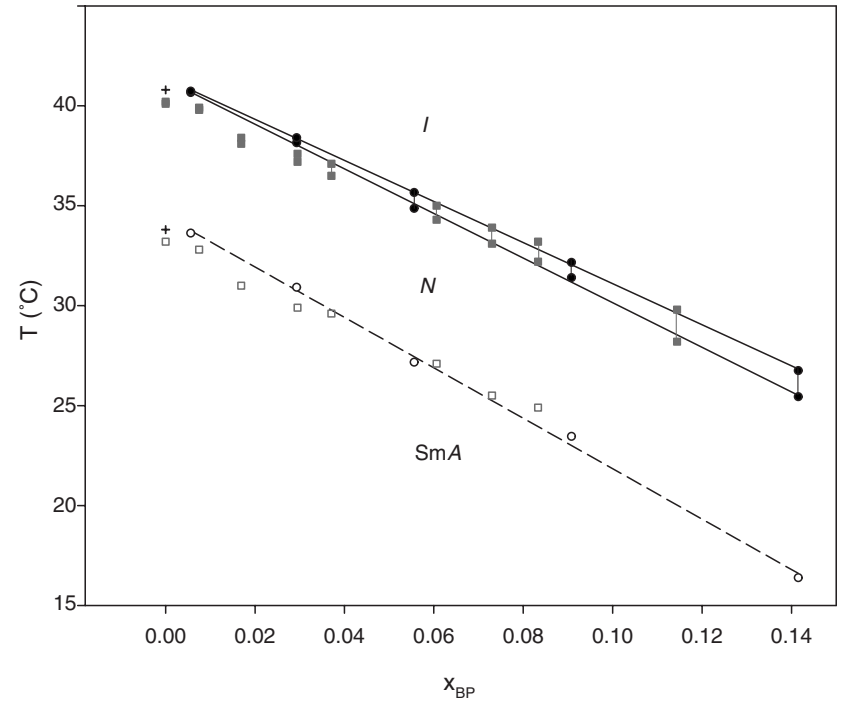

FIG. 1. Phase diagram of the system $8 \mathrm{CB}$ and biphenyl. The length of vertical line pieces indicates the width of the two-phase region. The solid and dashed lines are linear fits through the corresponding data points. The circles are present data, the crosses are literature values for pure $8 \mathrm{CB}$ [11], and the gray squares are from [21]. Full symbols represent a first-order phase transition and open symbols represent a continuous phase transition. The uncertainty on the temperatures is less than the symbol sizes.

\section{RESULTS AND DISCUSSION}

\section{A. Phase diagrams}

For the three mixture systems there are changes observed for the $N-I$ as well as for the $N-\operatorname{Sm} A$ transition temperature by adding the solutes. In Fig. 1 for $8 \mathrm{CB}+\mathrm{BP}$, in Fig. 2 for $8 \mathrm{CB}+\mathrm{CH}$, and in Fig. 3 for $8 \mathrm{CB}+W$ the phase transition temperatures are given as a function of the solute mole fraction $x$. For the first system the decrease of $T_{N-\operatorname{SmA}}$ with $x$ is somewhat stronger than for $T_{N-I}$. Our transition temperatures are in good agreement with literature values [21]. For 8CB $+\mathrm{CH}$ on the contrary $T_{N-I}$ decreases substantially faster with increasing $x$ than $T_{N \text {-SmA }}$.

With the exception of the largest mole fraction $x_{\mathrm{CH}}$ $=0.0722$, it turned out that the $\left(T_{N-I}\right.$ and $\left.T_{N-S m A}\right)$ transition temperatures for the $8 \mathrm{CB}+\mathrm{CH}$ mixtures prepared with batch 1 of $8 \mathrm{CB}$ (triangles in Fig. 2) were systematically lower than the ones prepared with the new batch 2 . We believe the lower transition temperatures for the other samples with $8 \mathrm{CB}$ of batch 1 are a consequence of sample handling. The first sample of $8 \mathrm{CB}+\mathrm{CH}\left(x_{\mathrm{CH}}=0.0722\right)$ was prepared by filling the cell with the required amounts of $8 \mathrm{CB}$ and $\mathrm{CH}$. Since from the subsequent measurements it turned out that $N-\operatorname{Sm} A$ transition was already strongly first-order, it was decided for the following measurements to stepwise reduce $x_{\mathrm{CH}}$ by evaporation of $\mathrm{CH}$ from the sample in the cell. This was done by heating the sample (in the open cell) with hot air from a heating gun. Four additional concentrations (with $x_{\mathrm{CH}}$ of $0.0104,0.0183,0.0368$, and 0.0467 ) were obtained in this way and subsequently measured. Apparently, by this procedure some (unknown) type of impurity must have been introduced to these samples. Recent testing of a small left-over

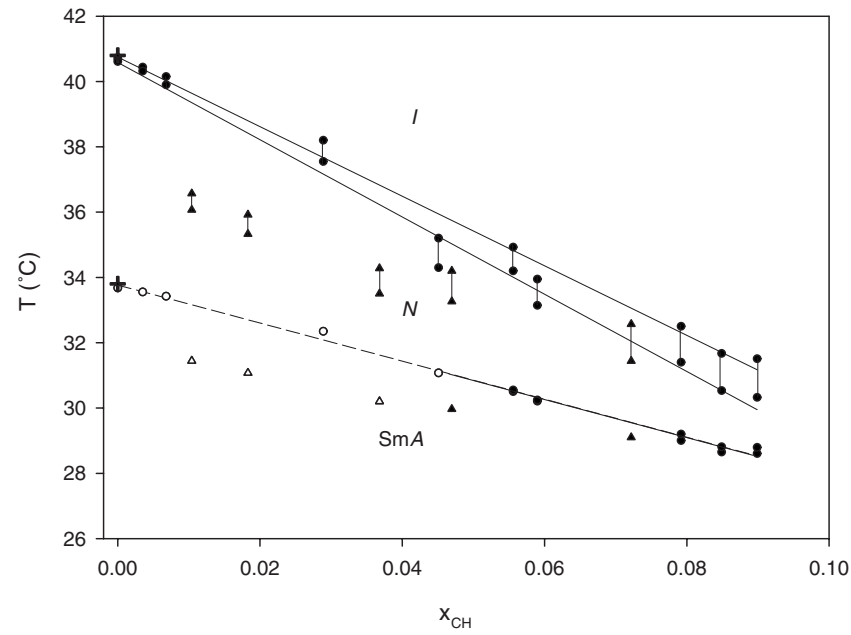

FIG. 2. Phase diagram for the system $8 \mathrm{CB}$ and cyclohexane. The length of the vertical line pieces indicates the width of the two-phase region. The triangles and circles are data corresponding with mixtures with $8 \mathrm{CB}$ of batch 1 and batch 2, respectively (see text). The crosses are for pure 8CB from [11]. Full symbols represent a first-order phase transition and open symbols a continuous one. The solid and dashed lines are linear fits through the corresponding data points of mixtures with $8 \mathrm{CB}$ from batch 2 . The uncertainty on the temperatures is less than the symbol sizes.

(in the original bottle) of the pure $8 \mathrm{CB}$ of batch 1 by polarizing microscopy resulted in transition temperatures consistent (within $0.2{ }^{\circ} \mathrm{C}$ ) with the well-established values of $T_{N-I}=40.7{ }^{\circ} \mathrm{C}$ and $T_{N-S m A}=33.7{ }^{\circ} \mathrm{C}[11]$. The transition temperatures for the new batch 2 are also in very good accordance with the values for pure $8 \mathrm{CB}$ [11]. For $8 \mathrm{CB}+\mathrm{CH}$ a second and a third series of samples was prepared with pure 8CB from batch 2 by adding small amounts of $\mathrm{CH}$ in steps. For the second series this is the $\mathrm{CH}$ mole fractions $x$ $=0.0451,0.0590,0.0849,0.0899$ and for the third series the

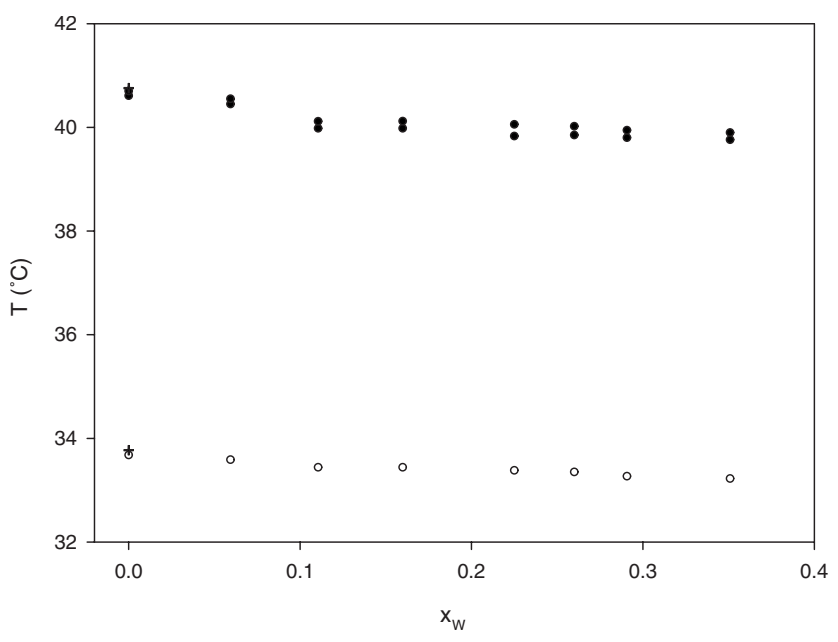

FIG. 3. Phase diagram of the system $8 \mathrm{CB}$ and water. The length of the short vertical line pieces indicates the width of the two-phase region. The circles are present data and the crosses are for pure $8 \mathrm{CB}$ from [11]. Full symbols represent a first-order phase transition and open symbols a continuous one. The uncertainty on the temperatures is less than the symbol sizes. 
$\mathrm{CH}$ mole fractions of $x=0.0035,0.0068,0.0289,0.0556$, 0.0792 . The different mole fractions in the cases of $8 \mathrm{CB}$ $+\mathrm{BP}$ and $8 \mathrm{CB}+W$ were all prepared by adding in steps small amounts of BP or W. For the second-order transitions of all three systems there was (within experimental resolution of about $10 \mathrm{mK}$ ) no indication of hysteresis between heating and cooling runs. For the first-order transitions, depending on the scanning rates, differences of 0.1 to $0.2{ }^{\circ} \mathrm{C}$ were observed in some cases (quite similar to what can be seen in pure systems). The phase diagram for $8 \mathrm{CB}+W$ in Fig. 3 shows a different behavior. After a small (about $0.8 \mathrm{~K}$ for $T_{N-I}$ and about $0.5 \mathrm{~K}$ for $T_{N-\mathrm{SmA}}$ ) decrease for mole fractions up to about 0.10 , the transition temperatures remain constant over the rest of the investigated $x$ range. An explanation for the approximately constant transition temperatures for the mixtures with $0.10<x_{W}<0.35$ was found after a visual inspection of six newly prepared mixture samples with different mole fractions of water. For mixtures with $x>0.10$, phase separation occurred: two phases, one mainly consisting of water and another one consisting mainly of $8 \mathrm{CB}$, were clearly visible. Thus, there exists an upper limit for the mole fraction of water that is soluble in $8 \mathrm{CB}$, and the liquid crystalline transitions observed for $x>0.10$ are those occurring in the $8 \mathrm{CB}$ rich phase with a nearly constant composition of $x$ around 0.10 . Inspection of different $8 \mathrm{CB}+\mathrm{BP}$ and $8 \mathrm{CB}$ $+\mathrm{CH}$ mixtures under the polarizing microscope did not give any indication of macroscopic segregation of $\mathrm{BP}$ or $\mathrm{CH}$ from 8CB.

An interesting question may arise regarding the distribution of the $\mathrm{BP}$ and $\mathrm{CH}$ impurity molecules between the $8 \mathrm{CB}$ liquid crystal host molecules, in particular in the smectic- $A$ phase. Although this question cannot properly be answered by macroscopic heat capacity measurements, visual observation or inspection under the polarizing microscope, our results indicate homogeneous distribution (at least on a mesoscopic scale) of $\mathrm{BP}$ and $\mathrm{CH}$ in the different samples studied. For $8 \mathrm{CB}+W$, however, clear macroscopic evidence was found that for $x_{W}>0.1$, water molecules are expelled from the host liquid crystal. In an x-ray diffraction experiment Rieker [22] showed for a monolayer $\operatorname{Sm} A$ and a monolayer $\mathrm{SmC}$ compound that the layer spacing increases with the addition of organic solvents ( $n$-decane, $n$-hexane, and benzene) to the host materials. The rate of this increase was considered an indication of the formation of an organic lyotropic, lamellar liquid crystal phase with the solvent intercalated between the smectic layers of the host liquid crystal. For $8 \mathrm{CB}$, exhibiting a $\operatorname{Sm} A_{d}$ layer spacing, results were less clear. Visual inspection of $8 \mathrm{CB}+n$-hexane and $8 \mathrm{CB}$ +benzene mixtures showed that the solvents were not uniformly distributed throughout the host liquid crystal. In both cases minimal or no swelling was observed. Thus, the question arises about the location in the 8CB host of the BP and $\mathrm{CH}$ molecules in our samples and whether intercalated impurity layers between the $\operatorname{Sm} A_{d}$ layers could be present. First, it should be noted that our studies are for rather dilute solutions $\left(x_{\mathrm{BP}}<0.15\right.$ and $\left.x_{\mathrm{CH}}<0.10\right)$ while in the x-ray experiment volume fractions of up to 0.8 were studied (in our cases volume fractions were more than an order of magnitude smaller). Although some layer swelling might also be present for $8 \mathrm{CB}+\mathrm{BP}$ and $8 \mathrm{CB}+\mathrm{CH}$ because the impurities

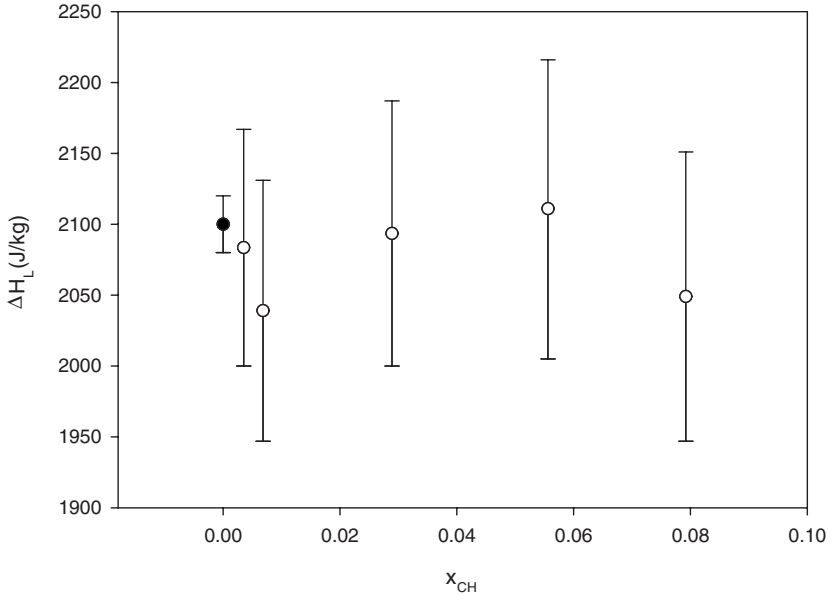

FIG. 4. Latent heats for the nematic to isotropic phase transition of the system $8 \mathrm{CB}$ and cyclohexane as a function of the mole fraction $x$ of cyclohexane. The value for pure $8 \mathrm{CB}$ (solid dot) is from [11].

might preferably intercalate between the layers (or inside the layers for BP?), there are, for mole fractions below 0.1 , not enough impurity molecules available to constitute a layer between each pair of smectic layers.

\section{B. The nematic-isotropic transition}

As observed in Fig. 1 for $8 \mathrm{CB}+\mathrm{BP}$ and in Fig. 2 for $8 \mathrm{CB}+\mathrm{CH}$ there is a rather strong decrease of $T_{N-I}$ with increasing mole fraction. Also for the (small) $x$ range where the amount of water in $8 \mathrm{CB}$ increases there is a decrease of the $N-I$ transition temperature with increasing $x$. This means that for these three cases the coefficient $E$ in Eqs. (13) and (14) must be positive. In Sec. II it was pointed out that if $E$ is a constant (and thus temperature independent) this would result in a first-order approximation in a $N-I$ latent heat that would not depend on the mole fraction of the solute. That this is, indeed, the case can be seen in Fig. 4 for a series of mixtures for the system $8 \mathrm{CB}$ with cyclohexane.

\section{The nematic-smectic- $A$ transition}

For the three systems $8 \mathrm{CB}+\mathrm{BP}$ (Fig. 1), $8 \mathrm{CB}+\mathrm{CH}$ (Fig. 2), and $8 \mathrm{CB}+\mathrm{W}$ (Fig. 3, in the range $x<0.10$ ), a decrease of $T_{N \text {-SmA }}$ is observed with increasing $x$. Considering Eqs. (12) and (19) it follows that (since all other parameters in these equations are positive) the parameter $A$ in Eq. (7) is positive.

For the five mixtures of $8 \mathrm{CB}$ and biphenyl it was found that the $N-\operatorname{Sm} A$ transitions are within experimental resolution, continuous with an upper limit for a latent heat (if present) of $\Delta H_{L}<2 \mathrm{~J} / \mathrm{kg}$. The specific heat capacity as a function of temperature near the transition was measured for all five mixtures and is depicted in Fig. 5. Also the effective critical exponent $\alpha$ was determined by plotting and fitting $\log _{10}\left(C-C_{p}\right)$ versus $\log _{10}|\tau|$. In Fig. 6 plots of $\log _{10}\left(C-C_{p}\right)$ versus $\log _{10}|\tau|$ for $T<T_{N-\mathrm{SmA}}$ are given with linear fits for the data in the range $-3.9<\log _{10}\left(C-C_{p}\right)<-2.5$. The effective critical exponent decreases from $\alpha=0.25$ for $x_{\mathrm{BP}}=0.0056$ to $\alpha=0.08$ for $x_{\mathrm{BP}}=0.1415$. 


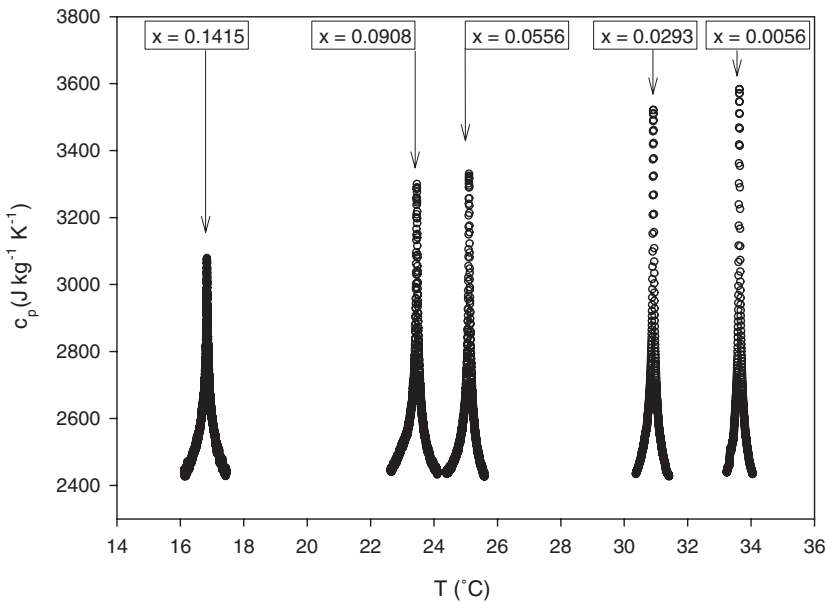

FIG. 5. Temperature dependence of the specific heat capacity for five mixtures of $8 \mathrm{CB}$ and biphenyl near the nematic to smectic- $A$ phase transition. The mole fractions $x=0.0293,0.0556$, and 0.1415 are heating runs and $x=0.0056$ and 0.0908 are cooling runs.

For mixtures of $8 \mathrm{CB}$ and cyclohexane the picture is completely different. For the concentrations below $x_{\mathrm{CH}}=0.046$, a continuous nematic to smectic- $A$ transition was observed within the experimental resolution $\left(\Delta H_{L}<2 \mathrm{~J} / \mathrm{kg}\right.$ for $x_{\mathrm{CH}}$ $=0.0035, x_{\mathrm{CH}}=0.0068, x_{\mathrm{CH}}=0.0104$, and $x_{\mathrm{CH}}=0.0183$; and $\Delta H_{L}<5 \mathrm{~J} / \mathrm{kg}$ for $x_{\mathrm{CH}}=0.0289, x_{\mathrm{CH}}=0.0368$, and $x_{\mathrm{CH}}$ $=0.0451)$. The effective critical exponent evolved from $\alpha$ $=0.33 \pm 0.03$ for $x_{\mathrm{CH}}=0.0035$ toward $\alpha=0.49 \pm 0.03$ for $x_{\mathrm{CH}}$ $=0.0451 . \log _{10}\left(C-C_{p}\right)$ versus $\log _{10}|\tau|$ plots for $T<T_{N-\operatorname{Sm} A}$ from which $\alpha$ values have been derived are shown in Fig. 7 for five concentrations. The $\log _{10}\left(C-C_{p}\right)$ versus $\log _{10}|\tau|$ data for the mixtures $x_{\mathrm{CH}}=0.0068$ and $x_{\mathrm{CH}}=0.028$ resulted in effective critical exponents of $\alpha=0.34 \pm 0.03$ and $\alpha$ $=0.43 \pm 0.03$, respectively. For clarity these data are not in-

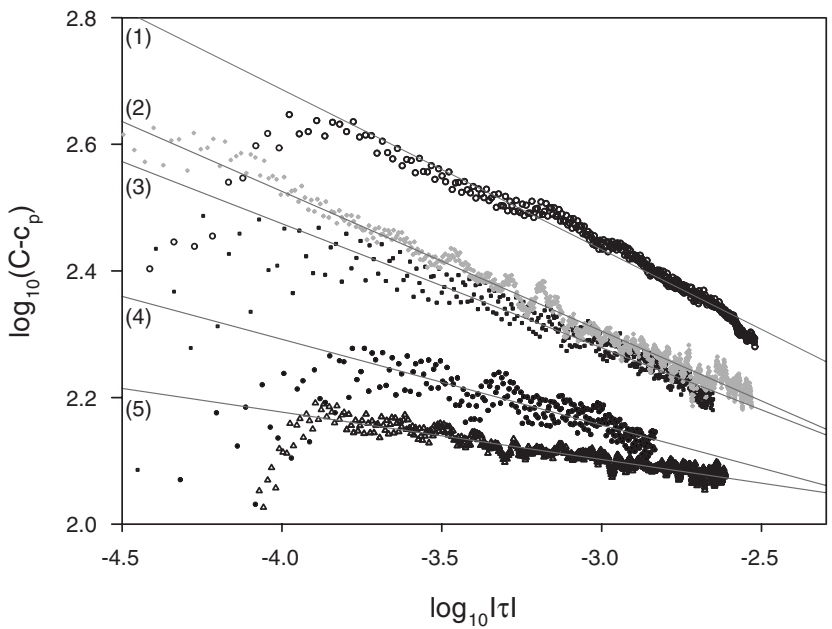

FIG. 6. Plots of $\log _{10}\left(C-C_{p}\right)$ versus $\log _{10}|\tau|$ below $T_{N \text {-SmA }}$ of the five mixtures of Fig. 5. The solid lines are linear fits (for -3.9 $\left.<\log _{10}|\tau|<-2.5\right)$ resulting in the indicated effective critical exponent $\alpha$ values. The uncertainty on $\alpha$ is \pm 0.03 . (1) $x=0.0056, \alpha$ $=0.25$; (2) $x=0.0293, \alpha=0.22$; (3) $x=0.0556, \alpha=0.20$; (4) $x$ $=0.0908, \alpha=0.14$; (5) $x=0.1415, \alpha=0.08$. The data (2), (3), and (5) are heating runs and (1) and (4) are cooling runs.

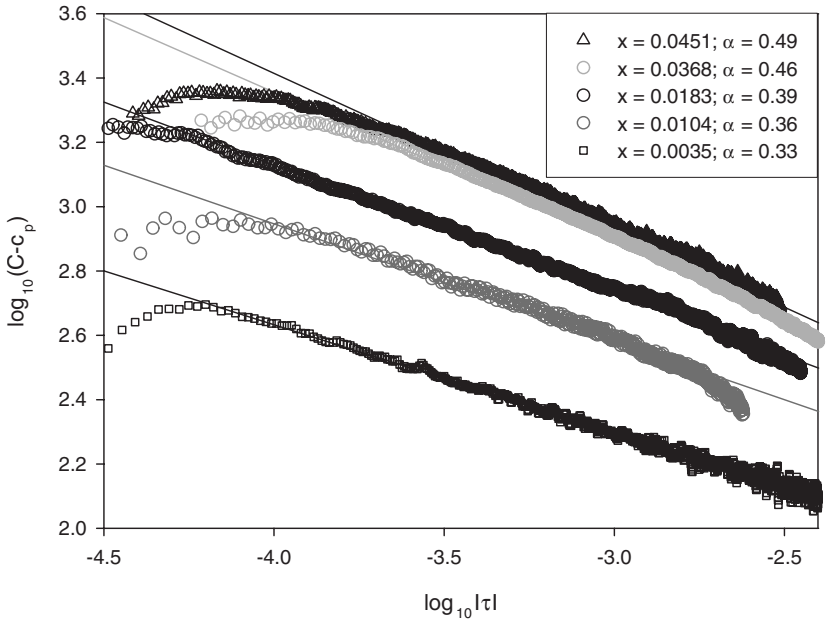

FIG. 7. Plots of $\log _{10}\left(C-C_{p}\right)$ versus $\log _{10}|\tau|$ below $T_{N \text {-SmA }}$ for five mixtures of $8 \mathrm{CB}$ and cyclohexane exhibiting a continuous phase transition. The mole fraction $x=0.0183$ is a heating run and $x=0.0451,0.0368,0.0104$, and 0.0035 are cooling runs. The solid lines are linear fits (for $-3.9<\log _{10}|\tau|<-2.5$ ) resulting in the indicated effective critical exponent $\alpha$ values. The uncertainty on $\alpha$ is \pm 0.03 .

cluded in Fig. 7. In Fig. 8 a plot of $\alpha$ as a function of $x_{\mathrm{CH}}$ shows clearly an evolution of $\alpha$ toward the tricritical value $\alpha=0.5$.

For mole fractions of cyclohexane higher than $x_{\mathrm{CH}}$ $=0.046$, a first-order $N-\operatorname{Sm} A$ transition is observed. The enthalpy as a function of temperature for the mixture with a mole fraction of cyclohexane equal to 0.0470 is plotted in Fig. 9. A finite latent heat of $17 \pm 5 \mathrm{~J} / \mathrm{kg}$ and a two-phase region of $\approx 15 \mathrm{mK}$ are clearly visible. In Fig. 10 it can be seen that for larger $x_{\mathrm{CH}}$ values the latent heat rapidly increases nonlinearly, reaching a value of $560 \pm 10 \mathrm{~J} / \mathrm{kg}$ for $x_{\mathrm{CH}}=0.0899$.

As already pointed out for the mixtures of $8 \mathrm{CB}$ and water in the phase diagram of Fig. 3 at first a slight decrease of the $N-I$ as well as of the $N-\operatorname{Sm} A$ phase transition temperature

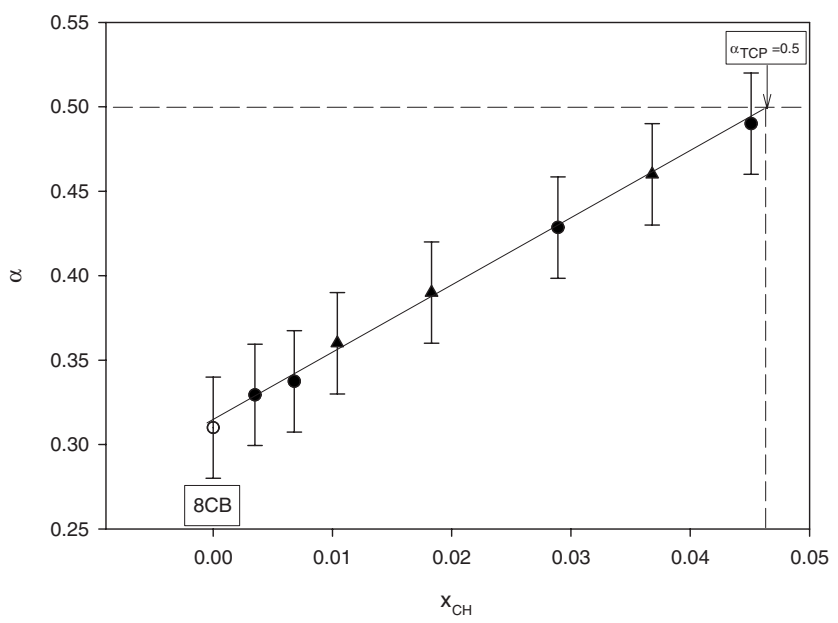

FIG. 8. Effective critical exponent $\alpha$ for the continuous phase transitions of the system $8 \mathrm{CB}$ and cyclohexane as a function of the mole fraction of cyclohexane. The value for pure $8 \mathrm{CB}$ is from [11]. 

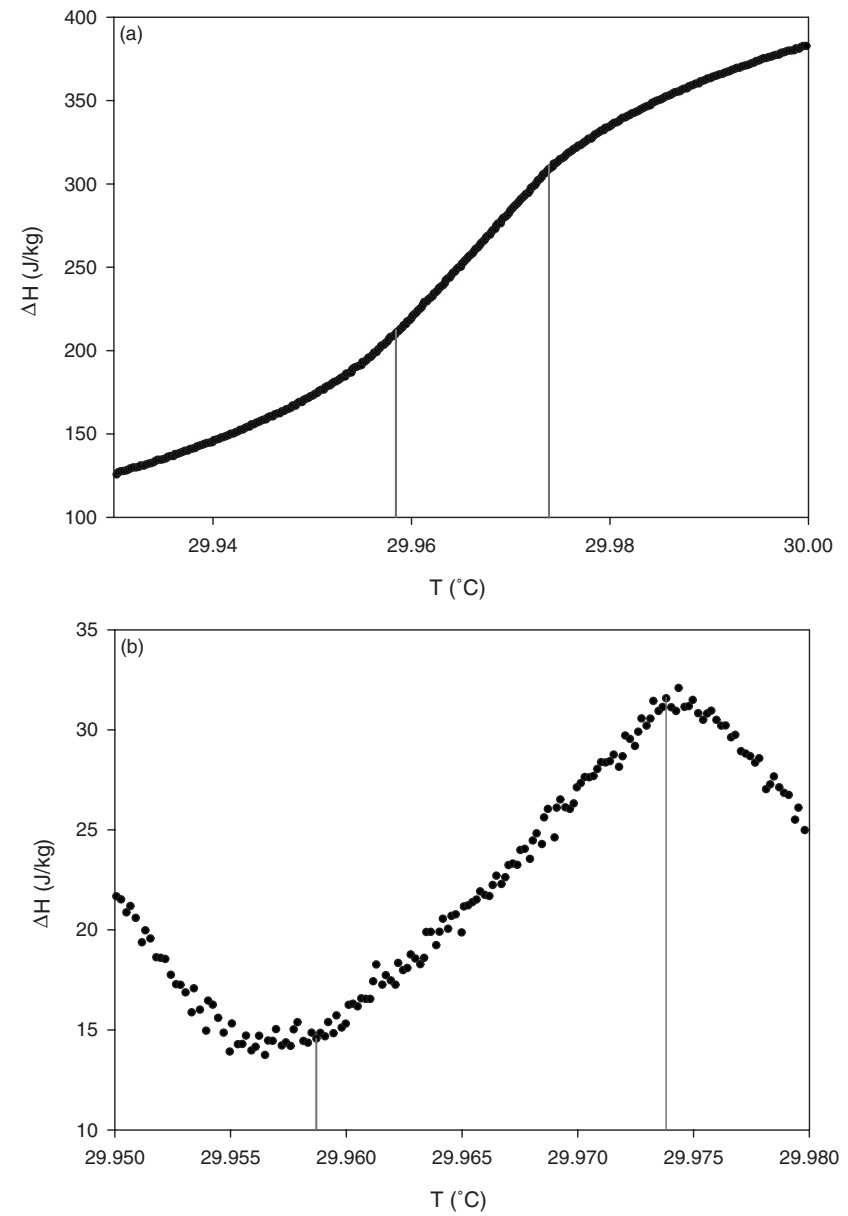

FIG. 9. Enthalpy near the $N-\operatorname{Sm} A$ phase transition of a mixture of $8 \mathrm{CB}$ and cyclohexane with mole fraction of cyclohexane $x_{\mathrm{CH}}$ $=0.0470$ with a linear background of (a) $3\left(T-T_{0}\right) \mathrm{J} / \mathrm{g}$ and (b) $8.8\left(T-T_{0}\right) \mathrm{J} / \mathrm{g}$ subtracted for clarity.

occurs over the range $0<x_{W}<0.10$, followed by nearly constant values for larger mole fractions. For these higher $x_{W}$ values, phase separation in a water-rich and a 8CB-rich phase took place. In all the mixtures, however, quite sharp $N-\operatorname{Sm} A$ heat capacity peaks could be observed, allowing the determination of effective critical exponent $\alpha$ values. A plot of the $\log _{10}\left(C-C_{p}\right)$ versus $\log _{10}|\tau|$ results for five mixtures $\left(x_{W}=0.0594, x_{W}=0.1105, x_{W}=0.1599, x_{W}=0.2606\right.$, and $x_{W}$ $=0.3509)$ is shown in Fig. 11. Equal values of $\alpha$ $=0.36 \pm 0.03$ were obtained for all the mixtures from $x_{W}$ $=0.1105$ to $x_{W}=0.3509$. For $x_{W}=0.0594$ an intermediate value $\alpha=0.32 \pm 0.03$ between this constant value of $0.36 \pm 0.03$ and the pure $8 \mathrm{CB}$ value of $0.31 \pm 0.03$ [11] was obtained.

The difference in the behavior of the three systems near the $N-\operatorname{Sm} A$ transition can be understood by looking at the changes in the width of the nematic ranges with mole fraction of the solute. In Fig. 12 it can be seen that with respect to the width of the $N$ range of pure $8 \mathrm{CB}$ it increases for $8 \mathrm{CB}+\mathrm{BP}$, decreases for $8 \mathrm{CB}+\mathrm{CH}$, and stays (after an initial small decrease) nearly constant for $8 \mathrm{CB}+W$ over the investigated $x$ range. For $8 \mathrm{CB}+\mathrm{CH}$ the narrowing of the nematic range results (at $x_{\mathrm{CH}} \approx 0.046$ ) in a crossover at a tricritical

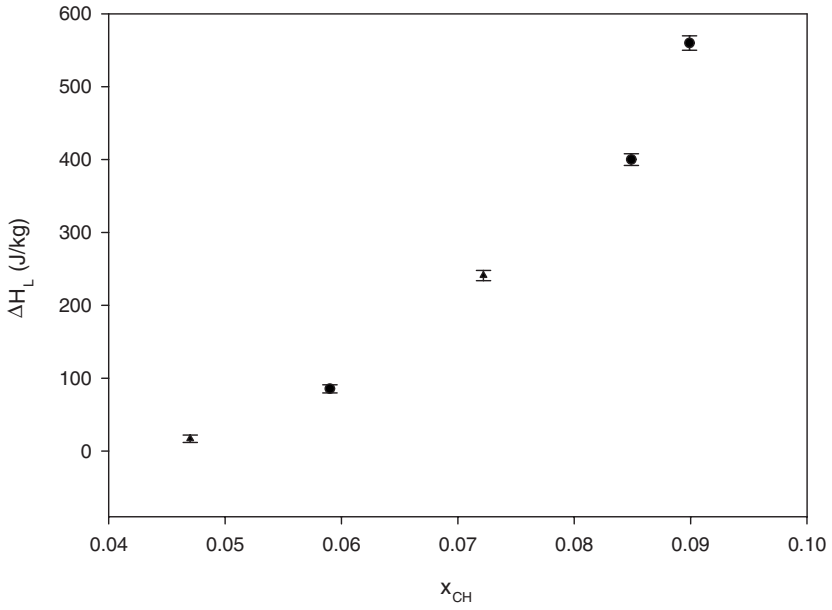

FIG. 10. Latent heat $\Delta H_{L}$ for the first-order $N$-SmA phase transitions of $8 \mathrm{CB}$ and cyclohexane as a function of mole fraction of cyclohexane $x_{\mathrm{CH}}$.

point from continuous transitions (at low $x_{\mathrm{CH}}$ values) to firstorder ones (at high $x_{\mathrm{CH}}$ ) with nonlinearly increasing latent heats with increasing $x_{\mathrm{CH}}$ (Fig. 10). For the continuous transitions the effective critical exponent $\alpha$ increases from the pure $8 \mathrm{CB}$ value of 0.31 to the tricritical value of 0.5 (see Fig. 8). This means that $\beta^{\prime \prime}$ of Eq. (11) changes sign at the tricritical point. As a consequence the parameter $D$ in that equation must be negative because for pure $8 \mathrm{CB}(x=0)$ one has $\beta^{\prime \prime}=\beta^{\prime}>0$. In Eq. (7) the fourth and seventh term can be combined to a single one with a coefficient $(C-D x)$ for the coupling term between the nematic and smectic- $A$ order parameters. Thus, because $D<0$ the addition of cyclohexane increases the coupling between these order parameters. The increasing width of the nematic range and the decreasing effective critical exponent $\alpha$ values for $8 \mathrm{CB}+\mathrm{BP}$, on the contrary, means that here $D$ in Eq. (11) has the opposite sign,

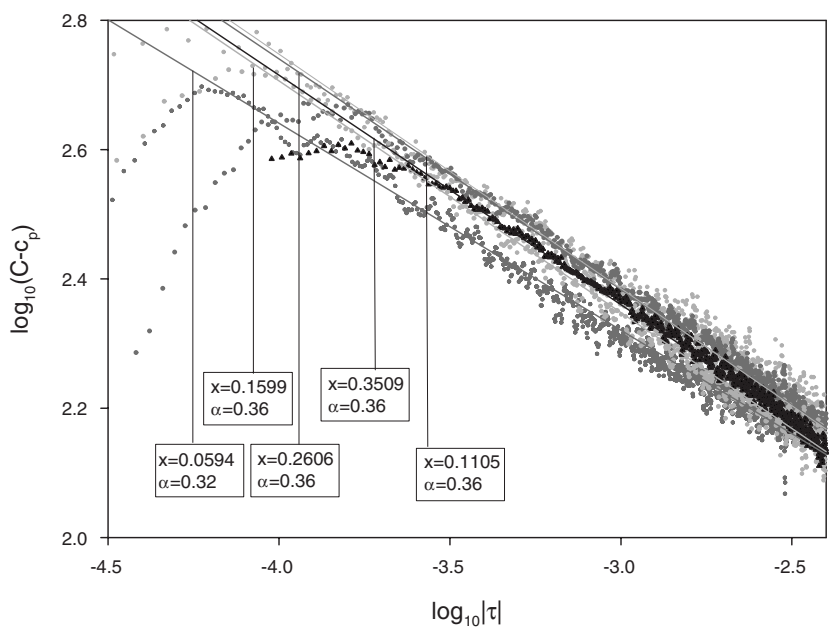

FIG. 11. Plots of $\log _{10}\left(C-C_{p}\right)$ versus $\log _{10}|\tau|$ below $T_{N \text {-SmA }}$ for five mixtures of $8 \mathrm{CB}$ and water exhibiting a continuous phase transition. The solid lines for $x_{W}=0.02603$ and $x_{W}=0.0594$ are linear fits for $-4.0<\log _{10}|\tau|<-2.4$, and for the other mixtures linear fits are for $-3.7<\log _{10}|\tau|<-2.4$, resulting in the indicated effective critical exponent $\alpha$ values. The uncertainty on $\alpha$ is \pm 0.03 . 


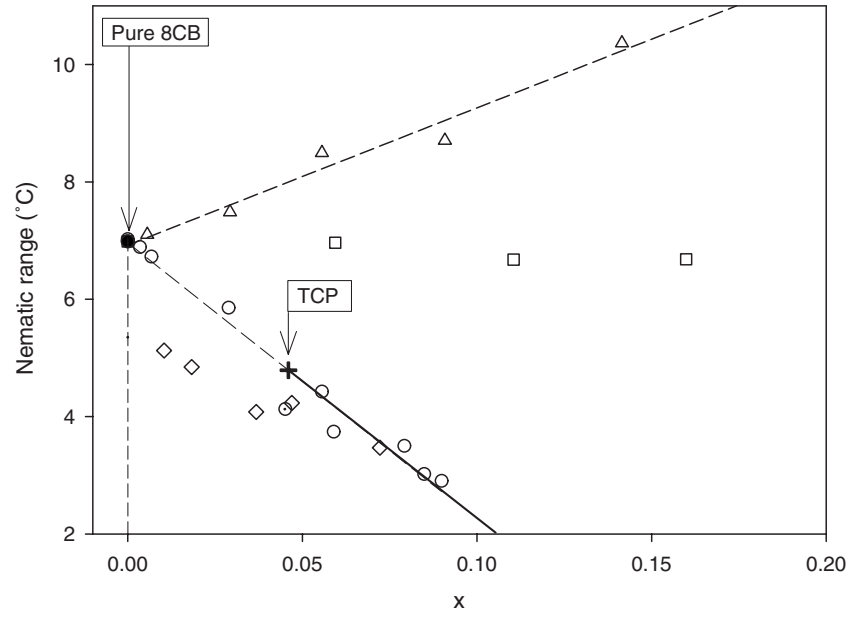

FIG. 12. The width of the nematic range as a function of the mole fraction of the nonmesogenic solutes. The solid dot is for pure $8 \mathrm{CB}$. The triangles are the mixtures of $8 \mathrm{CB}$ and biphenyl. The dashed line is a linear fit through these data. The squares are the results for $8 \mathrm{CB}$ and water. The mixtures of $8 \mathrm{CB}$ and cyclohexane are represented by the diamonds (batch 1 of $8 \mathrm{CB}$ ) and the circles (batch 2 of $8 \mathrm{CB}$ ). The dashed-solid line is a linear fit through the data of the mixtures of cyclohexane with $8 \mathrm{CB}$ of batch 2 . The tricritical point is indicated by the cross.

giving a decreasing coupling between the nematic and the smectic- $A$ order parameters, consistent with an increase in the nematic range. For the $8 \mathrm{CB}+W$ system the initial small reduction in nematic range and the slight increase in effective critical exponent $\alpha$ for mole fractions up to $x_{W} \approx 0.10$ needs also a (small) negative value for $D$ in Eq. (11). For larger $x_{W}$ values there is no further addition of water to the 8CB rich phase and in Eq. (11) Dx keeps the value it attained at $x_{W} \approx 0.10$, consistent with the constant width of the nematic range. Although, we based the above arguments on Eqs. (7) and (11), on the basis of Eq. (18) and the extension of Eq. (7) with a term $E S^{2} x$ (coupling between nematic order parameter and mole fraction) one reaches the same conclusions albeit with somewhat different coefficient values.

\section{SUMMARY AND CONCLUSIONS}

In this paper we reported on the effects of nonmesogenic solutes on the nematic to isotropic $(N-I)$ and nematic to smectic- $A(N-\operatorname{Sm} A)$ phase transitions of the liquid crystal octylcyanobiphenyl (8CB). Three different nonmesogenic impurities, being biphenyl (BP), cyclohexane $(\mathrm{CH})$, and water $(W)$, were added to $8 \mathrm{CB}$.

The effects on the $N-I$ transition were rather straightforward. For BP and $\mathrm{CH}$ a linear decrease of the $\mathrm{N}-\mathrm{I}$ transition temperature $T_{N-I}$ with the mole fraction of the solute was observed. For water as solute, $T_{N-I}$, at first slightly decreased for mole fractions of water up to $x_{W} \approx 0.10$ and stayed constant for higher values of $x_{W}$. From visual inspection of samples we found that at the higher mole fractions phase separation in a water rich and $8 \mathrm{CB}$ rich phase took place. In order to explain the linear decrease of $T_{N-I}$ one needs to extend the mean-field free energy density expression [Eq.
(1)] for the nematic order parameter $S$ with a coupling term between the solute mole fraction $x$ and the nematic order parameter $S$ of the form $E S^{2} x$, with $E$ a positive constant. It was also found that the $N-I$ transitions remained first-order ones with latent heats that did not change with mole fraction $x$ in the investigated range. This is to first-order consistent with a mean-field approximation and with $E$ independent of temperature.

As far as the nematic to smectic- $A$ transition temperatures $T_{N \text {-SmA }}$ are concerned the observations are similar to those for $T_{N-I}$. This means a linear decrease for $8 \mathrm{CB}+\mathrm{BP}$ and $8 \mathrm{CB}$ $+\mathrm{CH}$ and after a slight initial decrease (up to $x_{W} \approx 0.1$ ) a constant value for higher concentrations. To explain this decrease of $T_{N-S m A}$ in the free energy expansion [Eq. (3)] for the smectic- $A$ order parameter $\psi$ also a coupling term of the form $A \psi^{2} x$ ( $A$ a positive constant) with the mole fraction $x$ must be added. For the order of the transition as well as for the critical behavior of the specific heat capacity different results were obtained for the three systems. The $N-\operatorname{Sm} A$ transition for pure $8 \mathrm{CB}$ is continuous [11] and for all concentrations of biphenyl studied this transition remained continuous. The effective specific heat capacity critical exponent $\alpha$ decreased from $0.31 \pm 0.03$ for pure $8 \mathrm{CB}$ to $0.08 \pm 0.03$ for the highest mole fraction $\left(x_{\mathrm{BP}}=0.1415\right)$ of biphenyl studied. For mixtures of $8 \mathrm{CB}$ and cyclohexane, however, with increasing $x_{\mathrm{CH}}$ crossover from continuous to first-order transitions is observed at a tricritical point of the mole fraction of cyclohexane around $x_{\mathrm{CH}}=0.046$. For the continuous part of the phase transition line the value of the effective critical exponent $\alpha$ increased from $0.31 \pm 0.03$ for pure $8 \mathrm{CB}$ to 0.5 at the tricritical point. Along the first-order part of the transition line a nonlinear increase of the latent heat with increasing mole fraction was observed. For the $8 \mathrm{CB}$ and water system in all the mixtures, second-order $N-\operatorname{Sm} A$ transitions with quite sharp heat capacity peaks could be observed, allowing determination of effective critical exponent $\alpha$ values. For mixtures with $x_{W}>0.10$ a constant $\alpha=0.36 \pm 0.03$ was derived, for $x_{W}<0.10$ mixture, intermediate values between this $\alpha$ value and the pure 8CB $(\alpha=0.31)$ one were obtained.

The difference in the behavior of the three systems near the $N-\operatorname{Sm} A$ transition can be understood by looking at the changes in the width of the nematic ranges with mole fraction of the solute (see Fig. 12). For $8 \mathrm{CB}+\mathrm{BP}$ the width of the nematic range increases with increasing mole fraction of $\mathrm{BP}$, for $8 \mathrm{CB}+\mathrm{CH}$ it decreases with $x_{\mathrm{CH}}$, and for $8 \mathrm{CB}+W$ (after an initial small decrease up to $x_{W} \approx 0.10$ ) it stays constant over the investigated mole fraction range. Thus, one can conclude that depending on the type of impurity the coupling between the nematic and smectic- $A$ order parameters can either be reduced (wider nematic range) or increased (smaller nematic range) or kept constant (no changes in the nematic range). We have shown that in terms of the mean-field free energy density expansion of Landau-de Gennes in Eq. (3) the difference in behavior of the three systems could be accounted for by adding an additional coupling term between the nematic and the smectic- $A$ order parameters [see Eq. (7)] depending on the mole fraction of the solute with a coupling constant $D$ depending on the system. 


\section{ACKNOWLEDGMENTS}

One of the authors (J.T.) thanks M. A. Anisimov and C. W. Garland for valuable discussions. One of the authors (G.C.) thanks the Fund for Scientific Research Flanders. The authors also thank J. Leys for valuable suggestions and help with the preparation of this paper. This work was financially supported by the Fund for Scientific Research Flanders (Belgium) (FWO, Contract No. G.0125.03N) and by K. U. Leuven (Contract No. GOA-02).
[1] P. G. de Gennes and J. Prost, The Physics of Liquid Crystals (Clarendon, Oxford, 1993).

[2] G. Vertogen and W. H. de Jeu, Thermotropic Liquid Crystals, Fundamentals (Springer-Verlag, Berlin, 1988).

[3] J. Thoen, Int. J. Mod. Phys. B 9, 2157 (1995); J. Thoen, in Liquid Crystals in the Nineties and Beyond, edited by S. Kumar (World Scientific, Singapore, 1995), Chap. 2, p. 19.

[4] J. Thoen, in Physical Properties of Liquid Crystals, edited by D. Demus, J. Goodby, G. W. Gray, H. W. Spiess, and V. Vill (Wiley-VCH, Weinheim, 1997), pp. 208-232.

[5] C. W. Garland, in Liquid Crystals, Experimental Study of Physical Properties and Phase Transitions, edited by S. Kumar (Cambridge University Press, Cambridge, 2001), pp. 240294.

[6] B. Van Roie, J. Leys, K. Denolf, C. Glorieux, G. Pitsi, and J. Thoen, Phys. Rev. E 72, 041702 (2005).

[7] C. W. Garland and G. Nounesis, Phys. Rev. E 49, 2964 (1994).

[8] P. G. de Gennes, Solid State Commun. 10, 753 (1972); Mol. Cryst. Liq. Cryst. 21, 49 (1973).

[9] J. Thoen, H. Marynissen, and W. Van Dael, Phys. Rev. Lett. 52, 204 (1984); Mol. Cryst. Liq. Cryst. 124, 195 (1985).
[10] B. I. Halperin, T. C. Lubensky, and S. K. Ma, Phys. Rev. Lett. 32, 292 (1974).

[11] J. Thoen, H. Marynissen, and W. Van Dael, Phys. Rev. A 26, 2886 (1982).

[12] P. Jamée, G. Pitsi, and J. Thoen, Phys. Rev. E 67, 031703 (2003).

[13] A. Yethiraj and J. Bechhoefer, Phys. Rev. Lett. 84, 3642 (2000).

[14] M. A. Anisimov, P. E. Cladis, E. E. Gorodetskii, D. A. Huse, V. E. Podneks, V. G. Taratuta, W. van Saarloos, and V. P. Voronov, Phys. Rev. A 41, 6749 (1990).

[15] T. C. Lubensky, J. Phys. C 1, 151 (1975).

[16] L. Benguigui, Liq. Cryst. 25, 505 (1998).

[17] M. A. Anisimov, JETP Lett. 37, 11 (1983).

[18] S. DasGupta and S. K. Roy, Phys. Lett. A 288, 323 (2001).

[19] P. K. Mukherjee, J. Chem. Phys. 116, 9531 (2002).

[20] K. Denolf, B. Van Roie, C. Glorieux, and J. Thoen, Phys. Rev. Lett. 97, 107801 (2006).

[21] S. DasGupta, P. Chattopadhyay, and S. K. Roy, Phys. Rev. E 63, 041703 (2001).

[22] T. S. Rieker, Liq. Cryst. 19, 497 (1995). 